\title{
НАПРЯЖЕНИЯ В НЕПРЕРЫВНОЛИТОЙ ЗАГОТОВКЕ В ПРОЦЕССЕ ЕЕ ФОРМИРОВАНИЯ
}

\author{
А.С. Нурадинов, Л.Х-А. Саипова, Э.М. Балатханова, А.Н.Тепсаев \\ Грозненский государственный нефтяной технический университет \\ им. академика М.Д. Миллионщикова, г. Грозный, Россия
}

Качество заготовок непрерывного литья в значительной мере определяется не только конструкцией кристаллизатора, но и температурой разливаемой стали, а неравномерный теплоотвод от затвердевающей заготовки вызывает неодинаковое распределение температур в твердой корочке и различные деформационные свойства металла по высоте и поперечному сечению заготовки. Это приводит к развитию многочисленных поверхностных и внутренних дефектов, снижающих механические свойства литого металла, и сокращение срока службы металлоизделий из них.

Многие авторы связывают появление трещин в непрерывнолитой заготовке с ее напряженно-деформированным состоянием. Структура металла формируется в двухфазной зоне, которая представляет собой гетерогенную зону из растущих кристаллов с окружающим расплавом. С одной стороны двухфазная зона ограничивается твердой фазой, через которую происходит перенос тепла на молекулярном уровне (теплопроводностью), протекают диффузионные процессы, снижающие химическую неоднородность. С другой стороны она ограничена столбом жидкой стали, который оказывает ферростатическое давление. Затвердевающий объем в процессе формирования твердой оболочки, охлаждаясь, испытывает напряжения усадки. Закристаллизовавшиеся ранее внешние слои препятствуют усадке. В процессе выделения внутренней теплоты кристаллизации внешние слои разогреваются и расширяются, препятствуя усадке внутренних слоев. Таким образом, 
внутренние слои испытывают напряжения сжатия, а внешние соответственно напряжения расширения, под давлением которых оболочка сжимается и расширяется.

Однако кроме ферростатического давления жидкой стали и неравномерности распределения температурного поля, полузатвердевшая заготовка в МНЛЗ подвергается действию различных механических нагрузок, которые могут быть вызваны трением в кристаллизаторе, обжатием валков, несовпадением осей различных частей машины, а также возникших при разгибе и правке. К образованию трещин может привести любое из перечисленных условий.

Наибольший интерес с точки зрения причин образования трещин представляют главные параметры, влияющие на их возникновение - прочность, деформационная способность и скорость деформации металла при температурах затвердевания стали [1]. Необходимо, чтобы вследствие усадки стали, температурных напряжений и ферростатического давления суммарные напряжения, деформационная способность и скорость деформации не превышали их критических значений, при которых наступает разрыв металла [2].

Величины напряжений и деформаций, возникающих в оболочке заготовки, зависят от ее температуры, марки стали и других факторов. При значительных силах трения оболочка заготовки, находящаяся в кристаллизаторе, деформируется и даже может разрушиться, так как прочность ее при температурах, близких к температуре кристаллизации, сравнительно мала. Следствием деформации могут быть прорывы металла. В большинстве случаев они происходят на выходе из кристаллизатора, но зарождаются в результате предшествующего разрыва оболочки слитка в верхней части кристаллизатора.

Действующие в твердой оболочке заготовки растягивающие и сжимающие напряжения принимают различные значения в различных точках сечения твердой оболочки. Огромное влияние на возникновение и распределение 
напряжений в твердой оболочке оказывает равномерность продвижения фронта кристаллизации по направлению к технологической оси МНЛЗ. Неравномерность продвижения фронта кристаллизации обусловлена, прежде всего, разной скоростью кристаллизации отдельных участков, обусловленной, в том числе, неравномерным теплоотводом от поверхности. При наличии растягивающих напряжений в корке неравномерной толщины, в наиболее тонкой и нагретой части, концентрируется деформация, возникает изгибающий момент, стремящийся выгнуть эту часть оболочки внутрь корочки. При этом возможно открытие трещин в плоскости, перпендикулярной направлению продвижения фронта затвердевания, то есть в плоскости действия растягивающего напряжения.

Соответственно, основными видами напряжений, возникающих по толщине широких граней оболочки слитка при его затвердевании, являются: термические напряжения; растягивающие напряжения от действия ферростатического давления на узкие грани; напряжения от сил трения, обусловленные ферростатическим давлением на широкую грань при наличии перемещений оболочки вследствие ее усадки и при движении слитка относительно кристаллизатора; напряжения от момента изгиба оболочки на участках, прилегающих к углам.

Деформация металла весьма существенно влияет на допустимые напряжения в затвердевшей стали. В результате обработки экспериментальных данных предложены формулы для определения допустимого предела прочности в зависимости от температуры поверхности заготовки и температуры плавления заданной марки стали [3]:

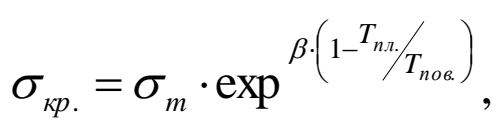

где $\sigma_{m}$ - напряжение вблизи температуры солидуса стали, равное по экспериментальным данным 7,5 - 8,0 МПа; $T_{n л}$ - температура плавления стали; $T_{\text {nов.- }}$ температура поверхности непрерывного слитка; $\beta$ - экспериментальный коэффициент, равный 6,88. 
При температуре, близкой к солидусу, значение допустимой деформации может быть определено по эмпирической формуле [3]:

$$
\varepsilon_{k p .} \approx 2,0 \cdot \tau^{-0,55},
$$

где $\tau$ - продолжительность затвердевания корочки.

Склонность к образованию трещин зависит от соотношения деформационной способности и прочностных свойств затвердевающей стали. Трещины всегда возникают в том случае, если прочность металла $\sigma$, относительная деформация $\varepsilon$ и скорость деформации $\dot{\varepsilon}$ определенного участка затвердевшего металла выше допускаемых, т.е. когда выполняются хотя бы одно из следующих неравенств [3]:

$$
\begin{aligned}
& \sigma>\sigma_{k p} ; \varepsilon>\varepsilon_{k p} ; \dot{\varepsilon}>\dot{\varepsilon}_{k p} ; \\
& \varepsilon=\frac{\Delta l}{l}=\frac{\alpha \cdot \Delta T \cdot l}{l}=\alpha \cdot \Delta T ; \quad \dot{\varepsilon}=\frac{\alpha \cdot \Delta T}{\tau} ; \quad \sigma=\varepsilon \cdot E
\end{aligned} .
$$

Здесь $\varepsilon$ - деформация; $\dot{\varepsilon}$ - скорость деформации; $\Delta l$ - усадка рассматриваемого участка заготовки; $l$ - протяженность участка заготовки; $\alpha$ - коэффициент усадки стали; $\Delta T$-перепад температур на рассматриваемом участке; $\tau$ - продолжительность охлаждения

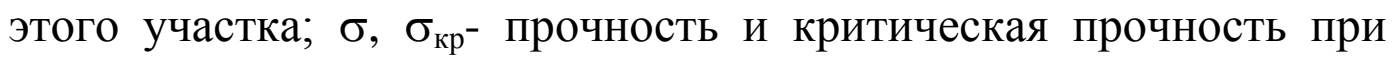
высоких температурах стали; Е - модуль упругости стали.

Таким образом, термонапряженное состояние корочки затвердевающей заготовки определяется прочностными и пластичными свойствами стали при высоких температурах, усадкой стали и образованием зазора между оболочкой заготовки и стенкой кристаллизатора, интенсивностью теплового потока и напряжениями в оболочке формирующейся заготовки после образования зазора.

Так как причиной возникновения термических напряжений считается только неравномерность температурного поля, то предполагается, что прочие воздействия на оболочку слитка (ферростатическое давление, усилия изгиба, вытягивания и пр.) 
можно исследовать отдельно с использованием принципа суперпозиции. Однозначного решения вопроса о степени влияния различных факторов на макроструктуру и качество поверхности непрерывнолитых заготовок по отдельным видам дефектов в многочисленных литературных источниках нет, так как каждая МНЛЗ представляет собой сложный агрегат со своей спецификой как в технологии разливки металла и конструкции зоны вторичного охлаждения, так и в обслуживании. В связи с этим совершенствование технологии непрерывной разливки идет в направлении создания моделей, описывающих технологические процессы с учетом большого числа технологических и конструктивных факторов [4].

В машине непрерывного литья твердая корка слитка формируется при высоких температурах достаточно медленно. Время формирования слитка велико по сравнению со временем релаксации напряжений. Сами приращения температурных напряжений в растущей корке слитка от изменения температурного поля при предельном переходе от одного момента времени к другому релаксируют практически мгновенно. Поэтому термонапряженное состояние корки слитка в процессе его затвердевания безынерционно соответствует температурному полю в данный момент времени [5].

В области моделирования термонапряжений и термодеформаций в настоящее время не существует единого подхода. Так в работе [6] рассматривается упругое приближение, в [7, 8] решается упруго-пластическая задача, в [9-11] используются модели вязкоупругого поведения материала. Существует также большое число различных постановок задач в рамках указанных основных подходов. Различия результатов в различных моделях зачастую весьма велики. Как показано в [11], напряжения на ранних этапах затвердевания стального слитка в кристаллизаторах машин непрерывного литья, рассчитанные по упругой модели, на два порядка выше напряжений, найденных по вязкоупругой модели. В то же время проведенные в [8] оценки 
времен релаксации напряжений указывают на возможность пренебрежения в этом случае вязкими эффектами. Ситуация еще более усугубляется отсутствием надежных данных о механических свойствах материалов при высоких температурах.

Как отмечается в $[10,11]$, в настоящее время наиболее широко используются два основных подхода к расчету термонапряжений в непрерывнолитом слитке, основанные на моделях упругопластического и вязкоупругого поведений материала.

При построении математической модели для расчета термических напряжений и деформаций учитывали, что формирование напряженного состояния происходит в условиях изменения толщины корки затвердевшего металла во времени, сложного характера теплообмена и т.д., т.е. в условиях сложного нагружения. Расчет напряжений и деформаций при наличии вязких и пластических эффектов требует в этом случае учета истории нагружения. Последнее достаточно полно можно осуществить только в рамках теории течения [12], в соответствии с которой процесс разбивался на последовательные этапы и задача решалась в приращениях напряжений и деформаций для поэтапного изменения нагружения.

В рассматриваемом случае затвердевшую часть слитка можно рассматривать как неограниченную вдоль координат $x$ и $z$ пластину переменной во времени толщины $h$, определяемой

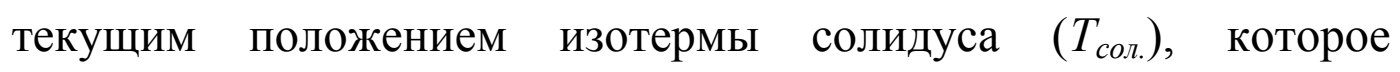

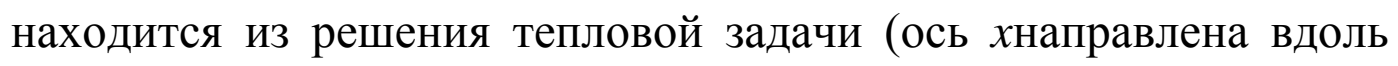
пластины, ось $z$ - по высоте, ось $y$ - поперек в направлении действия градиента температур).

Пренебрегая внешними механическими нагрузками по сравнению с термическими и учитывая, что температура пластины зависит лишь от координаты хв соответствии с [12] для любого $n$-го этапа нагружения, запишем:

$$
\begin{aligned}
& \Delta \sigma_{y}^{n}(x)=\Delta \sigma_{z}^{n}(x) \\
& \Delta \sigma_{x}^{n}=\Delta \sigma_{x y}^{n}=\Delta \sigma_{x z}^{n}=\Delta \sigma_{y z}^{n}=0 .
\end{aligned}
$$


Тогда уравнения равновесия при условиях (4) и выполняются тождественно, а уравнения совместности деформаций приобретают форму:

$$
\frac{\partial^{2} \Delta \varepsilon_{y}^{n}}{\partial x^{2}}=0, \frac{\partial^{2} \Delta \varepsilon_{z}^{n}}{\partial x^{2}}=0
$$

Соотношения, связывающие полные приращения деформаций, а также их упругие $\Delta \varepsilon^{l}$, пластические $\Delta \varepsilon^{p}$, вязкие $\Delta \varepsilon^{c}$ и термические $\Delta \varepsilon^{T}$ компоненты с приращениями напряжений, имеют вид [13] (индекс пдля сокращения записи опускаем):

$$
\begin{gathered}
\Delta \varepsilon_{x}=\Delta \varepsilon_{x}^{l}+\Delta \varepsilon_{x}^{p}+\Delta \varepsilon_{x}^{c}+\Delta \varepsilon^{T} \\
\left.\Delta \varepsilon_{z}=\Delta \varepsilon_{y}=\Delta \varepsilon_{y}^{l}+\Delta \varepsilon_{y}^{p}+\Delta \varepsilon_{y}^{c}+\Delta \varepsilon^{T}\right\} \\
\Delta \varepsilon_{x}^{l}=-\frac{2 v}{E} \Delta \sigma_{y}+2\left(\frac{v \sigma_{y}}{E^{2}} \frac{d E}{d T}-\frac{v \sigma_{y}}{E} \frac{d v}{d T}\right) \Delta T \\
\Delta \varepsilon_{y}^{l}=\frac{1-v}{E} \Delta \sigma_{y}-\left(\frac{(1-v) \sigma_{y}}{E^{2}} \frac{d E}{d T}-\frac{\sigma_{y}}{E} \frac{d v}{d T}\right) \Delta T \\
\Delta \varepsilon_{x}^{p}=\left(F_{\sigma}\left(\sigma_{i}, T\right) \Delta \sigma_{y}+F_{T}\left(\sigma_{i}, T\right) \Delta T\right) S_{x} \\
\Delta \varepsilon_{y}^{p}=\left(F_{\sigma}\left(\sigma_{i}, T\right) \Delta \sigma_{y}+F_{T}\left(\sigma_{i}, T\right) \Delta T\right) S_{y} ; \\
\Delta \varepsilon_{x}^{c}=v_{x} \Delta \tau ; \Delta \varepsilon_{y}^{c}=v_{y} \Delta \tau ; \\
\Delta \varepsilon^{T}=\alpha_{T}(\mathrm{~T}) \Delta \mathrm{T} ; F_{\sigma}=\frac{3}{2 \sigma_{i}}\left(\frac{1}{E_{k}}-\frac{1}{E}\right) ; F_{T}=\frac{3}{2 \sigma_{i}}\left(\beta+\frac{\sigma_{i}}{E} \frac{d E}{d T}\right) ; \\
\sigma_{i}=\sqrt{\left(\sigma_{y}^{2}+\sigma_{z}^{2}\right) / 2 ; S_{x}=-\frac{2}{3} \sigma_{y} ; \frac{\sigma_{y}}{3} .}
\end{gathered}
$$

Касательный модуль $E_{k}$ и коэффициент температурной податливости $\beta$ зависят от температуры и интенсивности напряжений $\sigma_{i}[13]$ и вычисляются по эмпирическим диаграммам растяжения $\sigma-\varepsilon$. Согласно теории ползучести Максвелла скорости ползучести в нашем случае имеют вид:

$$
v_{x}=\frac{s_{x}}{2 G \tau_{p}} ; v_{y}=\frac{s_{y}}{2 G \tau_{p}}
$$

и теории Нортона [13]:

$$
v_{x}=\frac{3}{2} B(T) \sigma_{i}^{m(T)^{-1}} S_{x} ; v_{y}=\frac{3}{2} B(T) \sigma_{i}^{m(T)^{-1}} .
$$


Учитывая то, что время релаксации в интересующем нас диапазоне является функцией температуры [13] $\tau_{p}(T)=9$. $10^{8} \exp (-0,01147)$ для стали, то можно аппроксимировать зависимости $B(T)$ и $m(T)$ в виде $m=5, \log B=0,0117(\mathrm{~T}-273)-21,69$. Решение задачи на каждом шаге осуществляется по методике [13]. Таким образом,

$$
\Delta \varepsilon_{z}=\Delta \varepsilon_{y}=\varepsilon_{0}+\chi_{0} x
$$

Неопределенные константы $\varepsilon_{0}$ и $\chi_{0}$ находятся из условий отсутствия внешних механических усилий и моментов:

$$
\int_{0}^{h} \Delta \sigma_{y} d x=0 ; \int_{0}^{h} \mathrm{x} \Delta \sigma_{y} d x=0 .
$$

При этом в (11) используются значения $\Delta \sigma_{y}$, найденные подстановкой (10) в (7):

$$
\Delta \sigma_{y}=\frac{\varepsilon_{0}+\chi_{0} x-\Delta \varepsilon^{T}-\Delta \varepsilon_{y}^{c}+\left(\frac{\sigma_{y}(1-v) d E}{E^{2} d T}-\frac{\sigma_{y} d v}{E d T}-F_{T} S_{y}\right) \Delta T}{\left(\frac{1-v}{E}+\frac{\sigma_{y}^{2} F_{\sigma}}{E}\right)} .
$$

После подстановки (12) в (11) интегралы можно взять численно. В результате на каждом шаге нагружения из (11) получаем систему линейных алгебраических уравнений, решая которую находим $\varepsilon_{0}$ и $\chi_{0}$ и далее по формуле (12) $\Delta \sigma_{y}$. Просуммировав полученное изменение напряжений с (12), характерних на предыдущих шагах нагружения, определяем полное напряжение на $n$-м этапе нагружения $\left(\sigma_{y}=\sigma_{y}+\Delta \sigma_{y}\right)$, после чего переходим к следующему шагу.

Устойчивость решения при учете вязких эффектов $\Delta \varepsilon^{c} \neq 0$ обеспечивается выбором временных шагов $\Delta \tau$ меньще характерних времен релаксации напряжений в наиболее горячих зонах слитка. Достаточно точное и устойчивое решение упругопластической задачи $\left(\Delta \varepsilon^{c}=0\right)$ получается при выполнении для каждой точки по толщине слитка условия $\Delta T \leq 10 K$.

Вывод: На основе проведенного анализа разработано программное обеспечение оценки изменения параметров 
формирования непрерывнолитой заготовки при различных режимах ее получения.

\section{Литература}

1. Кутателадзе С.С., Стырикович М.A. Гидродинамика газожидкостных систем. - М.: Энергия, 1976. - 296 с.

2. Еланский Г.Н. Разливка и кристаллизация стали. М: МГВМИ, 2010. - 192 c.

3. Алешко П.И. Механика жидкости и газа. - Харьков: Вища школа, 1977. $320 \mathrm{c}$.

4. Справочник по теплофизическим свойствам жидкостей и газов / Под ред. Варгафтика Н.Б. - М.: Наука, 1972. - 720 с.

5. Прандтль Л. Гидроаэромеханика. - М.: ИЛ, 1951. - 576 с.

6. Гуляев А.Г., Коминов С.В., Окороков Б.Н., Бакума С.С. Математическая модель процесса взаимодействия газовых струй с жидкой металлической ванной // Изв. ВУЗов. ЧМ. - №7. - 2000. - С. 20-22.

7. Монин А.С., Яглом А.М. Статистическая гидромеханика. - М.: Наука, 1965. Ч.1. $-640 \mathrm{c}$.

8. Якобше Р.Я., Козлова З.Л., Кучаев А.А., Дубоделов В.И., Фарук Саддики, Касьян Г.И. Влияние интенсивности электромагнитного перемешивания стали в кристаллизаторе МНЛЗ на структуру непрерывнолитой заготовки // Металлургическая и горнорудная промышленность. - 2006. - № 2. - C. 19-22.

9. Жильцов А.В., Касьян Г.И., Кучаев А.А., Петрушенко Е.И. Численное моделирование скорости жидкой стали в кристаллизаторе МНЛЗ при воздействии бегущего магнитного поля // Тезисы докладов Международной научно-технической конференции «50 лет в Академии наук Украины: ИЛП, ИПЛ, ФТИМС - прошлое, настоящее, будущее». 22-26 сентября 2008 года, г. Киев, Украина. - С. 131-135.

10. Сквориов А.А., Акименко А.Д. Теплопередача и затвердевание стали в установках непрерывной разливки. - М.: Металлургия. - 1988. - 143 с.

11. Борисов Б.Т. Теория двухфазной зоны металлического слитка. - М.: Металлургия, 1987. - 222 с.

12. Дымнич А.Х., Корниеи И.В. Основы теории подобия и физического моделирования. - Киев: Наш формат , 2016. - 172 с.

13. Еронько С.П., Быковских С.В. Физическое моделирование процессов внепечной обработки и разливки стали. - Киев: Техника, 1998. - 136 с. 